\title{
Motivación para viajar y satisfacción turística en función de los factores de personalidad
}

\author{
María García García* María de la Villa Moral Jiménez** \\ Universidad de Oviedo (España)
}

\begin{abstract}
Resumen: El tiempo libre es cada vez más amplio y el turismo supone una forma de ocuparlo como ocio, lo cual presenta diversas implicaciones algunas de las cuales son netamente psicosociales. Por tanto, la actividad turística constituye un fenómeno psicosocial digno de estudio. El objetivo de este estudio es analizar la relación entre la personalidad de los turistas y la motivación y la satisfacción turísticas, así como las diferencias en función del sexo, la edad y el nivel de estudios en las variables previamente mencionadas. Han participado 239 sujetos con edades comprendidas entre los 18 y los 82 años $(\mathrm{X}=33$; DT $=15,6)$ quienes, han respondido al Inventario de personalidad NEO-FFI, junto a las Escalas de motivación de la visita y Satisfacción turística. Asimismo, han indicado sus prioridades según el modelo de motivación turística de Pearce. En virtud de los resultados hallados, se ha comprobado la existencia de relación entre algunos rasgos de personalidad, diferentes motivaciones y la satisfacción turística. Se han obtenido diferencias estadísticamente significativas en motivación turística en función de la edad, aunque no en los indicadores de satisfacción. Asimismo, se ha confirmado que no existen diferencias significativas a nivel estadístico en función del nivel de estudios en las motivaciones turísticas, así como tampoco diferencias inter-género ni en motivación ni en satisfacción turística. Se abunda en la conveniencia de promover la investigación en esta temática que vincula la personalidad y el comportamiento turístico, dada su importancia a nivel psicosocial.
\end{abstract}

Palabras Clave: Turismo; Personalidad; Motivación; Satisfacción; Apertura a la experiencia.

\section{Motivation for traveling and tourist satisfaction in function of personality factors}

Abstract: Free time is growing and tourism is a way to spend it as leisure, which has various implications, some of which are purely psychosocial. Therefore, the tourism activity is a psychosocial phenomenon that is worth to study. The goal of the present work is to subject is to study the relationship between tourists personality, motivation and tourist satisfaction, as well as differences according to sex, age, and educational level in the aforementioned variables. The sample is made of 239 participants aged betweed 12 and 82 years $(\mathrm{X}=33, \mathrm{DT}=15.6)$ who have completed the NEO-FFI Personality Inventory, together with the Visibility Motivation Scales and Tourist satisfaction. They have also indicated their priorities according to Pearces tourism motivation model. Based on the results, it have been found relationships between some personality profiles, different motivations and tourist satisfaction. However, it were not found statiscally significant differences according to the level of studies class in tourism motivations, as well as no inter-gender differences in motivation or tourist satisfaction. It woud be interesting as a future prospect promote research in this field that links personality and tourist hebavior, according to its importance at the psychosocial level.

Keywords: Tourism; Personality; Motivation; Satisfaction; Opening to experience.

\section{Introducción}

Desde la vertiente aplicada de la investigación psicosocial no solo se ha procedido a aplicar los conocimientos a la solución de problemas sociales, en ámbitos diversos, tales como el mundo laboral, la salud, la educación y la publicidad, sino también al ámbito del turismo, el ocio y el tiempo libre. La ocupación del tiempo libre en la sociedad contemporánea es central en nuestras vidas y, una forma de emplearlo,

\footnotetext{
* Universidad de Oviedo (España); E-mail: UO246035@uniovi.es; https://orcid.org/0000-0001-7878-0578

** Universidad de Oviedo (España); E-mail: mvilla@uniovi.es; http://orcid.org/0000-0003-2421-9675
} 
justamente, consiste en hacer turismo (García, 1997). A nivel psicosocial se evidencia la pertinencia de analizar las necesidades a las que responde el tiempo libre y el ocio en la civilización postindustrial, así como de las actividades turísticas como máxima expresión del ocio (Moral, 2009). Específicamente, la conexión entre las actividades turísticas y la Psicología Positiva se constata en la literatura sobre el tema al promover ambas el bienestar de las personas (Garcés et al., 2018; López y Daniel, 2019).

Se justifica como marco conceptual la adopción de una perspectiva de análisis psicosocial en el estudio de la actividad turística y, específicamente de la demanda, en cuyo análisis se involucran factores sociodemográficos y geográficos, entre otros, si bien resulta fundamental entender de un modo comprehensivo la involucración de otros factores como los de índole psicosocial (motivaciones, personalidad, expectativas, necesidades, influencia social, etc.) que nos permiten entender cómo y por qué se comportan de un modo u otro las personas en lo relativo a la actividad turística. A su vez, la relevancia de este estudio para el turismo en los destinos visitados se vincula al propio valor aplicado del análisis psicosocial propuesto ya que las comunidades receptoras experimentarán beneficios inherentes al afianzamiento de su posición. En función de la satisfacción turística expresada y con una base motivacional, la tendencia a visitar tales destinos por turistas de uno u otro perfil de personalidad podría ser valorada, junto a otros factores, en estrategias de promoción y, propiamente, en las actividades ofertadas por proveedores turísticos, lo cual redundará en la fidelización del destino. La personalidad de los turistas resulta idónea como criterio de segmentación de mercados, de acuerdo con Medina-Ojeda et al. (2019). Asimismo, las actitudes, valores y demás características individuales de los potenciales turistas son variables psicosociales que aportan información de interés que pueden servir para adecuar la oferta a las propias expectativas vinculadas al destino y a las actividades de ocio, entre cuyos múltiples factores que determinan su comportamiento turístico, también se encuentran las propias características personológicas e identitarias de los turistas.

El tiempo libre es conceptualizado por Munné (1980) como aquel modo de darse el tiempo personal que es sentido como libre al dedicarlo a actividades auto-condicionadas al descanso y afirmarse a la persona individual y socialmente. Constituye un tiempo ocupado por aquellas actividades en las que predomina la libertad sobre la necesidad pues, en el caso de que se satisfagan algunas necesidades, estas resultan ser auto-creadas. Dentro de este tiempo libre se encuentra el ocio, que es descrito por Dumazedier (1974) como el conjunto de ocupaciones a las cuales la persona puede entregarse libremente, ya sea para descansar, divertirse o para desarrollar su formación desinteresada, su participación social voluntaria o su libre capacidad creadora, siempre y cuando estas se lleven a cabo después de haber cumplido con sus obligaciones profesionales, familiares y sociales. A su vez, el turismo comprende las actividades que realizan las personas en sus viajes y estancias, durante un periodo de tiempo superior a veinticuatro horas e inferior a un año, siempre y cuando estos se realicen en lugares distintos al de su entorno habitual, con fines de ocio, por negocios y otros motivos (Organización Mundial del Turismo, 1994). De este modo, desde la propia Organización Mundial del Turismo (2019a, p. 15) se define un destino turístico como "un espacio físico, con o sin una delimitación de carácter administrativo o analítico, en el que un visitante puede pernoctar. Es una agrupación (en una misma ubicación) de productos y servicios, y de actividades y experiencias, en la cadena de valor del turismo, y una unidad básica de análisis del sector".

La popularización de la actividad turística se asoció a la cultura del ocio en las primeras décadas del siglo XX y llegó a España con cierto retraso, si tomamos como referencia a los países vecinos (Pazos, 2017). Actualmente, se ha convertido en el segundo mayor destino del mundo por llegadas de turistas internacionales (82,7 millones) (Organización Mundial del Turismo, 2019b), de ahí que se haya incrementado el interés por esta temática. Como tal, constituye un fenómeno complejo multidimensional y transdisciplinar, de modo que la propia complejidad de la actividad turística se vincula a la diversidad de actores involucrados, así como a la propia dinámica de la actividad, de modo que, entre otros factores, también se atribuye a la esencia del comportamiento humano (González, 2018).

De acuerdo con Pinillos (1990), se describe primariamente el turismo como una afición a los viajes, a la curiosidad por ver tierras y gentes distintas a las propias. Resulta ser una actividad propulsada por un importante mecanismo psicofisiológico que reposa en última instancia en un hecho psicológico. No obstante, el turismo no se puede reducir únicamente a una motivación biológica elemental, también influyen motivaciones psicosociales al constituir una actividad con un eminente trasfondo socio-relacional (Moral, 2009).

Basándose en el modelo motivacional de Maslow (1943), Pearce (1982) propuso una adaptación incorporando el factor de la experiencia turística, de tal forma que determinó los siguientes niveles de motivación turística: a) Necesidad de relajación (descanso / actividad); b) Necesidad de estimulación (seguridad / emociones fuertes); c) Necesidades sociales (de familia y relaciones íntimas de amistad); $d$ ) Necesidades de autoestima (desarrollo personal, cultural, histórico, medioambiental), y e) Necesidades de autorrealización (búsqueda de la felicidad) (véase Colvin y Rutland, 2008). Años después, el mismo 
Pearce (1987) planteó una premisa de gran interés según la cual entre la Psicología y el turismo se puede establecer una relación de mutuo beneficio y de transferencia de conocimientos. Por un lado, el turismo permite estudiar en su ambiente natural algunos fenómenos psicosociales de relevancia como cambios de actitud, efectos del contacto intercultural, estereotipos, etc. Por otra parte, desde la Psicología Social se hacen contribuciones aplicadas a la actividad turística, como, por ejemplo: estudio de la satisfacción del consumidor, motivaciones para viajar, organización de servicios, etc. Constatada semejante relación simbiótica, es incuestionable que los turistas presentan diferentes motivaciones que responden, de forma sintetizada, en opinión de Araújo y Gosling (2017), a siete motivos recurrentes (p. 62): "1) búsqueda de autoconocimiento y crecimiento personal; 2) interés de vivir la diversidad cultural; 3) romper con la rutina y escapar de la realidad; 4) búsqueda de novedades; 5) búsqueda de aventuras y desafíos; 6) búsqueda de autenticidad y libertad; y 7) búsqueda de historias para contar".

Otro de los aspectos más importantes, junto con la motivación, que determinan la lealtad del turista es la satisfacción (Bigñé et al., 2001; Chi y Qu, 2008; Kozak y Rimmington, 2000; Yoon y Uysal, 2005; Yuksel et al., 2009). La satisfacción turística es definida por Oliver (1997) como un juicio, bien de naturaleza cognitiva, bien de carácter afectivo o emocional, que deriva de la experiencia del individuo con el servicio. De este modo, la imagen percibida de destino representa una variable mediadora del efecto de la motivación de viaje sobre la satisfacción turística (Olague et al., 2017). Y es que, por lo general, la propia anticipación de la conducta de viajar genera unas expectativas psicológicas positivas que deriva en sensaciones placenteras, de ahí la significación psicosocial del valor emocional del turismo (véase López y Daniel, 2019). Desde esa perspectiva de análisis psicosocial, en un sentido análogo, se plantea una asociación entre la satisfacción con un determinado destino y la propia personalidad del turista (Chon, 1992; Gountas y Gountas, 2007; Lee, 2013; Lin, et al., 2014).

En la demanda de servicios turísticos, además de la motivación y la satisfacción, existen otros rasgos que definen el comportamiento del turista, como la personalidad. Evidencia de ello la encontramos en que en la revisión de la literatura especializada varios autores se han interesado ya por este tema, aunque se debe resaltar que el intento de relacionar la elección de destino turístico con la personalidad es aún un tema novedoso (Ariffin et al., 2008; Gretzel et al., 2004; Lee, 2013; Leung y Law, 2010). Así, se han obtenido evidencias acerca de las posibles asociaciones entre los rasgos de personalidad de un individuo y su comportamiento turístico, en aspectos tales como la elección de un destino turístico (Leung y Law, 2010), la propia elección de actividades de ocio turístico (Kalmus, Realo y Siibak, 2011) e incluso la satisfacción y la fidelidad del turista (Gountas y Gountas, 2007), tal y como recoge Ojeda (2015).

Concretamente, Kalmus et al. (2011) utilizaron el Modelo de los Cinco Grandes factores de la personalidad para estudiar la influencia de esta en la búsqueda de información turística y el uso de Internet. También, se utilizó este mismo modelo para determinar la influencia de la personalidad en la elección del tipo de viaje o de un destino determinado (Abbate y Di Nuovo, 2013; Ariffin, et al. 2008) y, paralelamente, también ha sido empleado para conocer qué factores de la personalidad son los que influyen en la elección de un destino y de un tipo de viaje determinado, en función a su vez, de las motivaciones, tanto inherentes al individuo como las asociadas a atributos del destino turístico en sí (Ojeda, 2015).

Partiendo del esfuerzo de un grupo heterogéneo de investigadores (Digman y Takemoto-Chock, 1981; Goldberg, 1981; Norman, 1963), surgió el Modelo de los Cinco Factores de la Personalidad (Costa y McCrae, 1980) según el cual esta multiplicidad de rasgos resulta susceptible de agruparse en sólo cinco dimensiones: a) Neuroticismo: conjunto de rasgos vinculados a la inestabilidad emocional o a la tendencia a experimentar emociones negativas, tales como: miedos, tristeza, sentimientos de culpa o enojo (Widiger, 2009); b) Extraversión: agrupa aquellos rasgos que reflejan la tendencia a comunicarse con las demás personas, a ser asertivos, activos y verbalizadores (Wilt y Revelle, 2009); c) Apertura a la Experiencia o Apertura Mental: incluye un conjunto de rasgos que remiten a la capacidad de introspección o curiosidad intelectual, a una imaginación activa y a la sensibilidad estética (McCrae y Sutin, 2009); d) Amabilidad, Afabilidad o Tendencia al Acuerdo: referida a los rasgos que se asocian a la capacidad para establecer vínculos sociales, al altruismo y, a una marcada disposición a interesarse por los demás (Graziano y Tobin, 2009) y, finalmente, e) Responsabilidad, Escrupulosidad o Tesón: remite a aquellos rasgos ligados a la capacidad para identificar propósitos o metas claras, controlar impulsos, actuar, planificar, organizar y llevar a cabo proyectos e ideas (Roberts et al., 2009). Específicamente, el interés de estudio en los que vincula a variables personológicas la elección de destinos turísticos y la motivación para viajar es evidenciado por McCrae y Costa (2003), quienes inciden en que la personalidad constituye uno de los factores explicativos de mayor relevancia. De ahí la elección de su modelo explicativo y de evaluación de la personalidad centrado en su descripción no en sus causas, aunque, evidentemente, se han desarrollado múltiples teorías de la personalidad desde distintos enfoques o perspectivas, ya sea 
el de rasgos, como este o perspectivas más biologicistas, fenomenológicas o cognoscitivas, entre otras. En este sentido, Costa y McCrae (1990) sostienen que el Modelo de los Cinco Grandes ha contribuido a la modelización de la estructura de la personalidad, de acuerdo con el análisis de Cloninger (2003).

Respecto al análisis de la motivación turística, variable de interés en este estudio, según Andrade (2012) aproximadamente el 96\% de los turistas declaran que desconectar, cambiar de aires y salir de la rutina eran sus principales motivos para realizar turismo, en este caso de tipo rural. Asimismo, relajarse y descansar, también, son elegidos en segunda posición como lo más buscado con un $95,4 \%$. El 90\% de los encuestados se siente motivado por disfrutar estando en contacto con la naturaleza, como forma de aliviar el estrés y la tensión acumulados del día a día, así como para disfrutar del tiempo libre con la familia y/o amigos (ambos con 87,5\%). Por el contrario, ir a un destino de moda, contar las aventuras del viaje, dar a conocer el lugar a amigos y familiares o conocer gente nueva, acumulan los porcentajes más bajos (entre 55 y 60\%). Por su parte, Moreno et al. (2012) determinaron como prioritarias las motivaciones turísticas relacionadas con evadirse de la rutina diaria, descansar y aliviar el estrés y la tensión, seguidas de la búsqueda de diversión y entretenimiento y, en menor medida, la realización de actividades de recreo y deportivas. De las motivaciones centradas en el conocimiento se resaltan el conocer lugares nuevos y descubrir diferentes culturas. Las motivaciones sociales más destacadas son las de disfrutar y compartir tiempo con familia y amigos. Dentro de las motivaciones de reconocimiento social y prestigio, visitar lugares confortables con buenos hoteles y restaurantes, resulta un aspecto con importancia relativamente elevada. Sin embargo, apenas despiertan interés las motivaciones relacionadas con ir a lugares visitados con anterioridad por amigos o que estén de moda.

Relacionado con lo anterior, en el caso de la satisfacción turística se estima que depende de dos factores: el factor humano y la valoración de accesibilidad a los servicios (Devesa y Palacios, 2005). En este sentido, Laguna y Palacios (2009) encontraron como primer y principal indicador de satisfacción la amabilidad y el trato a los empleados, resultados parecidos a los obtenidos con anterioridad por Hartline et al. (2003). Se considera que hay un conjunto de indicadores de la satisfacción que no se relacionan con la motivación y se denominan satisfactores generales, correspondiéndose con aquellos relacionados con la disponibilidad de los recursos, ya sea información vía Web o a través de folletos, instalaciones de ocio o accesibilidad, así como también factores humanos e interpersonales, tales como el trato recibido, la hospitalidad, la profesionalidad de los empleados y la seguridad (Devesa et al., 2008, 2010a, 2010b). En relación con ello, el visitante con una motivación más de tipo gastronómico y cultural estacional puntúa más en satisfacción las visitas guiadas, el tráfico y los aparcamientos, valoran la profesionalidad de la hostelería, la oferta de alojamiento, el entorno natural y la seguridad ciudadana. A su vez, el visitante con una motivación monumental de cercanía se relaciona con mayores puntuaciones de satisfacción en conservación del patrimonio; los visitantes hedonistas y de descanso son los que puntúan en más satisfacción en tranquilidad, horarios, presencia de puntos de información y espacios especiales para turistas, entre otros indicadores (Devesa et al., 2010b).

Respecto al estudio de la personalidad, Ojeda (2015) asevera que las personas con una motivación de descansar presentan un perfil de personalidad mayor en agradabilidad y menor en apertura a la experiencia y búsqueda de sensaciones; aquellas cuya motivación es escapar de la rutina puntúan más en agradabilidad y apertura a la experiencia; cuando la motivación es familiar el turista se caracteriza por no buscar sensaciones y, finalmente, cuanto más grande es el deseo de disfrutar de la experiencia mayor puntuación obtienen en los rasgos de personalidad extraversión, agradabilidad, apertura a la experiencia y búsqueda de sensaciones.

También se han estudiado las influencias positivas y negativas que reciben las motivaciones según los perfiles de personalidad, de acuerdo con Ojeda (2015), afirmando lo siguiente: a) la motivación para descansar está potenciada por la agradabilidad y penalizada por la apertura a la experiencia y la búsqueda de sensaciones; $b$ ) la motivación a escapar de la rutina recibe influencias positivas de la agradabilidad y negativas de la extraversión; c) la motivación familiar solamente resulta afectada de forma negativa por la búsqueda de sensaciones; y, $d$ ) la motivación de disfrutar de la experiencia solo se ve influenciada de manera positiva por la extraversión, agradabilidad, apertura a la experiencia y búsqueda de sensaciones. Asimismo, se afirma que: a) quienes conceden mayor importancia a disfrutar del sol y la playa presentan mayores niveles de extraversión, agradabilidad, apertura a la experiencia y búsqueda de sensaciones; $b$ ) las personas que prefieren conocer el lugar de destino e interactuar con sus gentes son los que presentan un perfil de personalidad más extravertido, con rasgos de agradabilidad, apertura a la experiencia y búsqueda de sensaciones; $c$ ) los que se decantan por visitar atractivos culturales y naturales ostentan mayor nivel de responsabilidad y apertura a la experiencia; y, asimismo, 
d) las personas que se interesan por practicar deportes presentan menores niveles de neuroticismo y mayores de apertura a la experiencia.

Finalmente, respecto a las variables sociodemográficas, Moreno Gil et al. (2012) comprueban que según la edad, los turistas menores de 34 años se sienten más motivados por la diversión que aquellos con edades superiores, destacando a su vez que las personas mayores de 54 años son las más motivadas por la interacción social. Posteriormente, se afirma que a mayor edad mayor importancia se les concede a las motivaciones familiares y de descanso y, en niveles más bajos a disfrutar de la experiencia (Ojeda, 2015).

Una vez fundamentado lo anterior, el objetivo general de este estudio es determinar la relación entre la motivación para la elección de destino turístico y la satisfacción turística con los rasgos de personalidad. Como objetivos secundarios se propone analizar la relación que vincula la motivación y la satisfacción turísticas. Asimismo, se analizarán las diferencias en motivación y satisfacción turísticas en función del sexo, del rango de edad y del nivel de estudios.

Se plantean las siguientes hipótesis:

H1: Se propone una relación entre la motivación turística y los rasgos de personalidad en el sentido de que las personas extravertidas y aquellas con tendencia a la amabilidad presentan motivación turística socio-relacional y las que destaquen por la apertura experiencial y mental se decantan por la motivación cultural. H2: La satisfacción turística difiere en función de los rasgos de personalidad, de tal forma que, las personas neuróticas manifiestan menor satisfacción que las personas extravertidas, abiertas, afables y responsables. H3: Entre la motivación y la satisfacción turísticas se constata una relación positiva en el sentido de que a mayor motivación turística general mayor satisfacción.

H4: Se espera que haya diferencias inter-género en motivación y satisfacción turísticas. De esta forma, las mujeres mostrarán más motivación turística de descanso y monumental, con satisfacción media y, los hombres tenderán a mostrar más motivación turística por la búsqueda de nuevas experiencias, con una satisfacción mayor. H5: Existen diferencias entre la motivación turística y la satisfacción, en función de la edad, las personas más jóvenes presentan mayor motivación sobre la búsqueda de nuevas experiencias con satisfacciones también más elevadas y, las personas más adultas muestran una motivación más hedonista y de descanso, de tipo social-relacional, monumental y gastronómica, con una satisfacción que puede oscilar entre media y buena.

\section{Metodología}

\section{Participantes}

La muestra se compone de un total de 239 participantes residentes principalmente en el Principado de Asturias (65.5\%), aunque también en otras comunidades autónomas como Castilla y León, Cantabria, El País Vasco y la Comunidad Valenciana, que han sido seleccionados mediante un muestreo opinático intencional de tipo no probabilístico.

Según el sexo, un 59,4\% $(\mathrm{n}=142)$ son mujeres y un $40,6 \%$ son hombres $(\mathrm{n}=97)$. Atendiendo al rango de edad, un 59,4\% $(\mathrm{n}=142)$ tienen de 18 a 29 años y un 40,6\% $(\mathrm{n}=97)$ presentan 30 años (incluidos) en adelante. El rango de edad se sitúa entre los 18 y los $82(\mathrm{X}=33$; DT $=15,6)$.

En lo referente a la condición socio-económica, dependiente esta del rango de ingresos por unidad familiar, encontramos que un 40,2\% $(n=96)$ se adscribe a la clase media $(24.868,80 €-34.816,20 €)$ y que un $33,9 \%(\mathrm{n}=81)$ ha cursado hasta Bachillerato y presentan estudios universitarios un $28,9 \%(\mathrm{n}=69)$.

\section{Instrumentos de medida}

Para la realización del presente trabajo instrumentos de medida, los cuales detallaremos a continuación.

En primer lugar, se han tenido en cuenta variables sociodemográficas tales como: el sexo, la edad, la condición socioeconómica (según Ayala, 2018), y el nivel de estudios.

En segundo lugar, para la evaluación del constructo personalidad, se ha aplicado el NEO-FFI, que es la versión reducida del Inventario de Personalidad NEO PI-R. Esta recoge los 60 mejores elementos de la versión extendida permitiendo evaluar, igualmente, la personalidad según los cinco grandes factores, aunque, sin desglosarlos en facetas: a) Neuroticismo. Facetas: ansiedad, hostilidad, depresión, ansiedad social, impulsividad, vulnerabilidad; b) Extraversión. Facetas: cordialidad, gregarismo, asertividad, actividad, búsqueda de emociones, emociones positivas; c) Apertura. Facetas: fantasía, estética, sentimientos, acciones, ideas, valores; d) Amabilidad. Facetas: confianza, franqueza, altruismo, actitud conciliadora, modestia, sensibilidad a los demás y, finalmente, e) Responsabilidad. Facetas: competencia, orden, sentido del deber, necesidad de logro, autodisciplina, deliberación. El Alpha de Cronbach obtenido en este estudio alcanzó un valor de 0.759 . 
Los participantes de este estudio han valorado sus motivaciones para viajar asociadas a sus experiencias turísticas habituales. Para evaluar aspectos motivacionales se solicitó a los participantes que ordenasen según su prioridad el total de los cinco indicadores de la pirámide de la Motivación Turística de Pearce, indicándolo mediante los números del uno al cinco siendo, el número 1 el más prioritario y el 5 al que menos prioridad le otorgamos. Asimismo, se ha aplicado la Escala sobre la Motivación de la Visita (Fernández, Laguna y Palacios, 2010). Se incluyen variables que comprendían tanto factores internos como externos, siguiendo la distinción que estableció Crompton (1979) entre motivos de arrastre y de empuje. Está conformado por 17 ítems en una escala de medición de tipo Likert que hemos adaptado a 5 puntos: 1 totalmente en desacuerdo a 5 totalmente de acuerdo. Consta de 5 factores: Motivaciones hedonistas y de descanso; Motivo social relacional; Motivaciones monumentales y gastronómicos; Búsqueda de nuevas experiencias; y, finalmente, Factores de conveniencia y utilitarios. El alpha de Cronbach obtenido por los autores de la escala alcanzó un valor de 0.749 y en este estudio 0.700 .

Finalmente, se ha empleado una escala sobre Satisfacción Turística (Fernández et al., 2010). Está conformado por 26 ítems en una escala de medición tipo Likert que hemos adaptado a 5 puntos: 1 muy insatisfecho a 5 muy satisfecho. La escala integra información procedente de seis aspectos relacionados con la valoración del destino turístico: infraestructura viaria, infraestructura hotelera, infraestructura de restauración, trato recibido, conservación del patrimonio natural y cultural e información y accesibilidad turística. Las respuestas de los participantes responden a una demanda turística efectiva habiéndose analizado sus experiencias turísticas. El alpha de Cronbach obtenido por los autores de la escala alcanzó un valor de 0.810 , obteniéndose 0.892 en este estudio.

\section{Procedimiento}

La recogida de datos se realizó a través de un formulario elaborado en la plataforma online de Google, que estuvo activo entre el 25 de febrero y el 27 de abril de 2019. Se difundió mediante el procedimiento de bola de nieve compartiéndolo a través de WhatsApp a personas conocidas, pidiéndoles, a su vez, que lo reenviasen ellos a sus allegados. Como estrategia paralela, se procedió a difundirlo, a su vez, a través de redes sociales, con el objetivo de reclutar aún más sujetos. Las redes sociales utilizadas fueron Facebook, principalmente, y Discord.

La primera parte del cuestionario hacía referencia al consentimiento informado, donde se explicaban los fines de la investigación, además se informaba del carácter anónimo de sus respuestas. Antes de continuar, las personas debían dar su consentimiento en esta primera parte. Además, se indicaba también que sólo se podía rellenar una vez por persona y que no debían retroceder y cambiar sus respuestas una vez que hubiesen completado cada sección correspondiente. Una vez completado, los participantes accedían a cubrir la información solicitada.

\section{Diseño}

El estudio llevado a cabo sigue un diseño transeccional correlacional multivariado donde los datos se recabaron en un solo momento. Han sido seleccionados mediante un muestreo no probabilístico intencionado en el que se analizaron varias variables simultáneamente.

\section{Análisis de los datos}

En primer lugar, a objeto de contrastar las hipótesis en las que se planteaban una relación entre los rasgos de personalidad y la satisfacción turística y entre aquella y la motivación turística se han llevado a cabo correlaciones de Spearman, dada la naturaleza de las variables. A su vez, se han correlacionado los factores de motivación y de satisfacción turística mediante el estadístico de Pearson que es una medida lineal entre dos variables aleatorias cuantitativas.

Para estudiar la relación entre la motivación turística y variables sociodemográficas, como el sexo, la edad y el nivel de estudios se han empleado comparaciones de medias. Específicamente, se han empleado pruebas t de comparaciones de medias para muestras independientes para contrastar la hipótesis en la que se plantean diferencias significativas en motivación y satisfacción turística en función del sexo y de la edad, calculando el tamaño del efecto d de Cohen. En el caso concreto del nivel de estudios, se ha utilizado el estadístico Anova de un factor, comprobando posteriormente entre qué niveles de la variable se encontraban las diferencias significativas mediante la prueba de Bonferroni.

El software empleado para realizar el análisis de los datos estadísticos es el paquete estadístico SPSS. 


\section{Resultados}

Siendo objeto de interés en la primera hipótesis planteada el estudio de la relación entre las motivaciones turísticas y los rasgos de personalidad, como se expone en la Tabla 1, se ha obtenido una correlación significativa entre el factor Extraversión de personalidad y la búsqueda de nuevas experiencias, las motivaciones monumentales y gastronómicas, sociorrelacionales y con los factores de conveniencia y utilitarios, aunque con valores bajos. A su vez, también resulta significativa la correlación entre la dimensión de Apertura y la búsqueda de nuevas experiencias, al igual que el factor de Amabilidad con las motivaciones monumentales y gastronómicas con valores igualmente bajos. Asimismo, se ha obtenido una correlación significativa, pero baja, entre el factor Responsable y las motivaciones hedonistas y de descanso, así como con la monumental y gastronómica.

Se constata que el factor Neurótico no correlaciona significativamente con ninguna de las motivaciones turísticas. Por su parte, la personalidad Extravertida no correlaciona con la motivación hedonista y de descanso. El rasgo de personalidad de Apertura no correlaciona con las motivaciones hedonistas y de descanso, sociorrelacionales ni monumentales y gastronómicas, pero tampoco con los factores de conveniencia y utilitarios. La personalidad Amable, no correlaciona con las motivaciones hedonistas y de descanso, socio-relacionales ni con la búsqueda de nuevas experiencias. Finalmente, el rasgo de Responsabilidad no correlaciona con la motivación socio-relacional y tampoco con la búsqueda de nuevas experiencias ni con los factores de conveniencia y utilitarios.

Tabla 1: Relación entre las motivaciones turísticas y los factores de personalidad

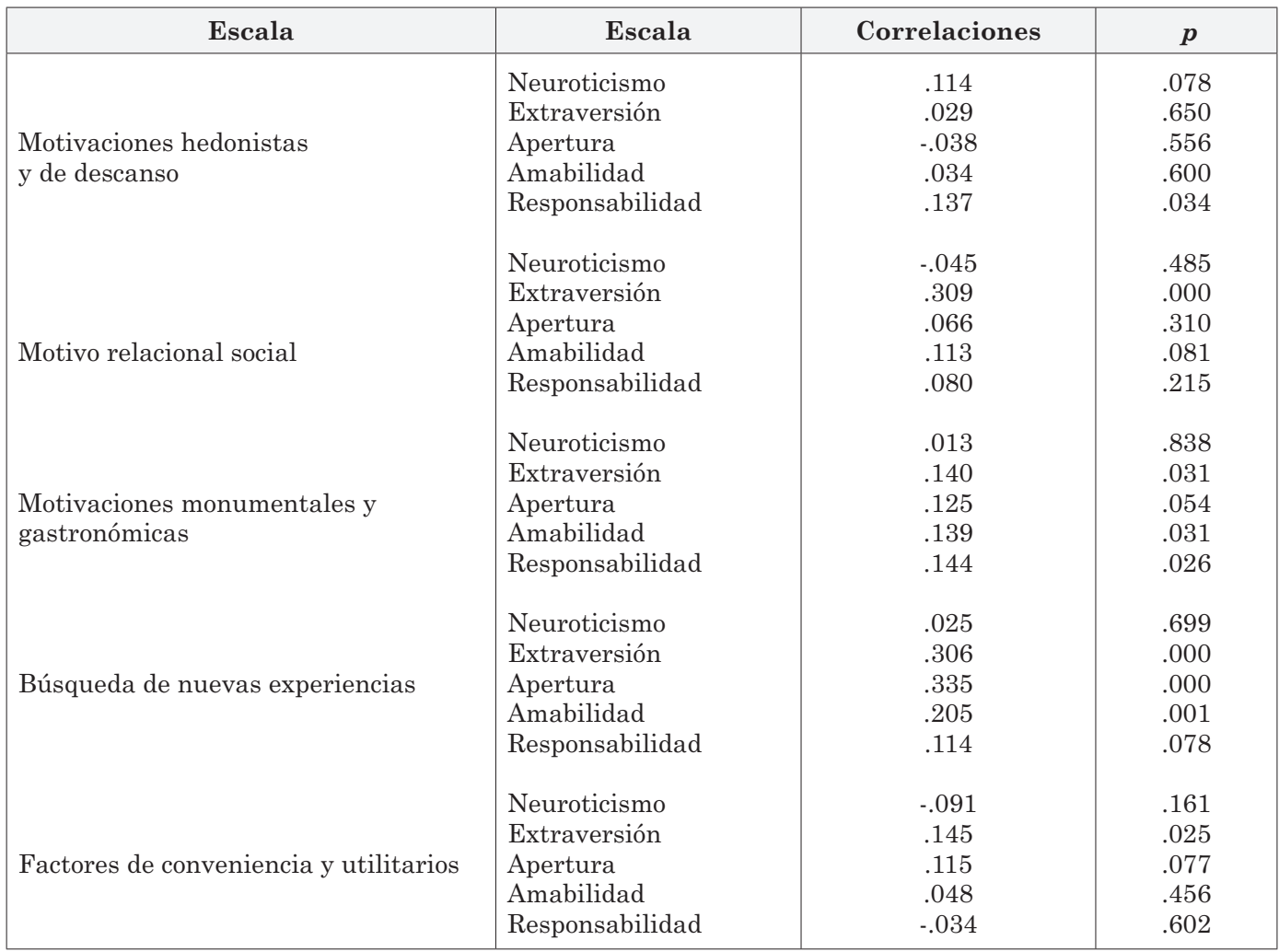

Con el objeto de estudiar la relación entre la satisfacción turística y los diferentes rasgo de personalidad, se comprueba que únicamente se da una correlación positiva significativa entre la satisfacción turística y el perfil de personalidad de Apertura $(r=.348)$. Sin embargo, no se da correlación entre 
la satisfacción turística y los rasgos de Neuroticismo, Extraversión, Amabilidad y Responsabilidad (véase Tabla 2).

Tabla 2: Relación entre la satisfacción turística y los factores de personalidad

\begin{tabular}{|l|c|c|}
\hline \multicolumn{1}{|c|}{ Escala } & Correlaciones & $\boldsymbol{p}$ \\
\hline Neuroticismo & .022 & .731 \\
Extraversión & .028 & .665 \\
Apertura & .348 & .022 \\
Amabilidad & .086 & .184 \\
Responsabilidad & .001 & .993 \\
\hline
\end{tabular}

Partiendo del interés en estudiar la relación entre la satisfacción y las motivaciones turísticas, se confirma la existencia de una correlación significativa entre la satisfacción turística y las motivaciones sociorrelacionales $(\mathrm{r}=.330)$, monumentales y gastronómicas $(\mathrm{r}=.291)$ y con la búsqueda de nuevas experiencias $(\mathrm{r}=.219)$. No obstante, no se ha hallado correlación entre la satisfacción turística y las motivaciones hedonistas y de descanso, y tampoco con los factores de conveniencia y utilitarios.

Se constata que no existen diferencias inter-género en los indicadores de motivación turística (Motivaciones hedonistas y de descanso, Motivo relacional social, Motivaciones monumentales y gastronómicas, Búsqueda de nuevas experiencias y Factores de conveniencia y utilitarios) ni en satisfacción turística general, tal y como se expone en la Tabla 3.

Tabla 3: Diferencias en la satisfacción y motivaciones turísticas en función del sexo

\begin{tabular}{|l|c|c|c|c|c|c|}
\hline \multicolumn{1}{|c|}{$\begin{array}{c}\text { Hombre } \\
\text { (n= 97) }\end{array}$} & \multicolumn{2}{c|}{$\begin{array}{c}\text { Mujer } \\
\text { (n=142) }\end{array}$} & t & $\boldsymbol{p}$ \\
\hline \multicolumn{1}{|c|}{ Variables dependientes } & $\boldsymbol{M}$ & $\boldsymbol{D T}$ & $\boldsymbol{M}$ & $\boldsymbol{D T}$ & & \\
\hline Satisfacción turística & 94.680 & 11.519 & 95.556 & 11.623 & -.574 & .566 \\
\hline $\begin{array}{l}\text { Motivaciones hedonistas y de } \\
\text { descanso }\end{array}$ & 23.443 & 3.511 & 23.838 & 3.201 & -.900 & .369 \\
\hline Motivo relacional social & 9.752 & 1.859 & 10.119 & 1.976 & -1.444 & .150 \\
\hline $\begin{array}{l}\text { Motivaciones monumentales y } \\
\text { gastronómicas }\end{array}$ & 10.134 & 2.206 & 10.091 & 2.231 & .145 & .885 \\
\hline $\begin{array}{l}\text { Búsqueda de nuevas } \\
\text { experiencias }\end{array}$ & 12.010 & 2.152 & 12.352 & 1.928 & -1.283 & .201 \\
\hline $\begin{array}{l}\text { Factores de conveniencia y } \\
\text { utilitarios }\end{array}$ & 4.814 & 2.228 & 4.964 & 2.118 & -.528 & .598 \\
\hline
\end{tabular}

Se planteó que se obtendrían diferencias en función de la edad en motivación y satisfacción turísticas. Estas diferencias por edad se hallaron en la motivación sociorrelacional y la búsqueda de nuevas experiencias, cuyos tamaños del efecto son bajo-medio y medio, respectivamente. Asimismo, no se confirma la existencia de diferencias en la satisfacción turística ni en las motivaciones hedonistas y de descanso, monumentales y gastronómicas ni en los factores de conveniencia y utilitarios en función de este indicador sociodemográfico (véase Tabla 4). 
Tabla 4: Diferencias en la satisfacción y motivaciones turísticas en función de la edad

\begin{tabular}{|c|c|c|c|c|c|c|c|}
\hline \multicolumn{3}{|c|}{$\begin{array}{l}\text { Edad joven } \\
(n=142)\end{array}$} & \multicolumn{2}{|c|}{$\begin{array}{l}\text { Edad adulta } \\
\quad(\mathrm{n}=97)\end{array}$} & \multirow[t]{2}{*}{$\mathbf{t}$} & \multirow[t]{2}{*}{$p$} & \multirow[t]{2}{*}{$\begin{array}{l}\text { d de } \\
\text { Cohen }\end{array}$} \\
\hline Variables dependientes & $M$ & $D T$ & $M$ & $D T$ & & & \\
\hline Satisfacción turística & 95.626 & 11.435 & 94.577 & 11.785 & .688 & .492 & \\
\hline $\begin{array}{l}\text { Motivaciones hedonistas } \\
\text { y de } \\
\text { descanso }\end{array}$ & 23.873 & 3.206 & 23.391 & 3.498 & 1.098 & .273 & \\
\hline Motivo relacional social & 10.246 & 1.838 & 9.567 & 2.009 & 2.701 & .007 & .352 \\
\hline $\begin{array}{l}\text { Motivaciones } \\
\text { monumentales y } \\
\text { gastronómicas }\end{array}$ & 10.084 & 2.261 & 10.144 & 2.160 & -.204 & .838 & \\
\hline $\begin{array}{l}\text { Búsqueda de nuevas } \\
\text { experiencias }\end{array}$ & 12.647 & 1.861 & 11.577 & 2.095 & 4.147 & .000 & .540 \\
\hline $\begin{array}{l}\text { Factores de conveniencia y } \\
\text { utilitarios }\end{array}$ & 4.901 & 2.337 & 4.907 & 1.882 & -.020 & .984 & \\
\hline
\end{tabular}

Asimismo, se planteó que se obtendrían diferencias en función del nivel de estudios en las motivaciones turísticas, sin embargo, no se ha confirmado la significación de las mismas (véase Tabla 5).

Tabla 5: Diferencias en las motivaciones turísticas en función del nivel de estudios

\begin{tabular}{|c|c|c|c|c|c|c|c|}
\hline \multirow{2}{*}{$\begin{array}{c}\text { Variables } \\
\text { dependientes }\end{array}$} & \multicolumn{5}{|c|}{ Medias } & \multirow{2}{*}{$\mathbf{F}$} & \multirow{2}{*}{$\mathbf{p}$} \\
\hline & ESO & Bach. & Grado & FP & Universitarios & & \\
\hline $\begin{array}{l}\text { Motivaciones } \\
\text { hedonistas y de } \\
\text { descanso }\end{array}$ & 21.885 & 23.851 & 23.871 & 24.857 & 23.681 & 1.277 & .262 \\
\hline Motivo relacional social & 9.625 & 10.086 & 9.580 & 11.000 & 10.058 & 1.261 & .271 \\
\hline $\begin{array}{l}\text { Motivaciones } \\
\text { monumentales y } \\
\text { gastronómicas }\end{array}$ & 10.312 & 10.000 & 9.354 & 11.000 & 10.376 & 1.303 & .250 \\
\hline $\begin{array}{l}\text { Búsqueda de nuevas } \\
\text { experiencias }\end{array}$ & 11.062 & 12.469 & 11.677 & 12.142 & 12.304 & 1.407 & .203 \\
\hline $\begin{array}{l}\text { Factores de } \\
\text { conveniencia y } \\
\text { utilitarios }\end{array}$ & 4.687 & 4.580 & 4.903 & 4.857 & 5.202 & .613 & .745 \\
\hline
\end{tabular}

\section{Discusión}

Los aspectos novedosos aportados en este trabajo son los relativos al estudio de la relación entre los perfiles de personalidad de los turistas, la motivación para viajar y la satisfacción turística, así como la búsqueda de diferencias en las motivaciones turísticas en función del sexo, la edad y el nivel de estudios del turista.

Entre las aportaciones concretas de este estudio destaca la constatación de que los turistas más extravertidos y con mayor apertura intelectual presentan motivaciones para viajar asociadas a la búsqueda de nuevas experiencias y no así motivaciones hedonistas o de descanso, de ahí que modalidades 
de turismo experiencial les podrían resultan atractivas. A su vez, el turismo patrimonial se vincularía a personas con apertura intelectual. Para valorar la aplicación de estos hallazgos en los destinos conviene ser conscientes de la interrelación de múltiples factores determinantes de la elección de destino, pero sugiriendo que la personalidad es uno de los que determinan la motivación para viajar y el propio comportamiento turístico.

Específicamente, conviene destacar la relación mejor establecida con valores correlaciónales superiores a .30 se da entre la búsqueda de nuevas experiencias y los rasgos de Apertura y Extraversión, así como entre esta última y los motivos sociorrelacionales. En concreto, también se ha hallado una relación entre la Extraversión y las motivaciones monumentales y gastronómicas, y con los factores de conveniencia y utilitarios. A su vez, el factor de Apertura se vincula a la búsqueda de nuevas experiencias, mientras que el de Amabilidad se asocia a las motivaciones monumentales y gastronómicas con valores bajos. Asimismo, se ha constatado que el factor de personalidad calificado como Neurótico no correlaciona significativamente con ninguna de las motivaciones turísticas.

Concretamente, Ojeda (2015) destaca que las personas con motivación para descansar presentan personalidad Agradable, mientras que en el presente estudio se encuentra que las personas con dicha motivación manifiestan una tendencia al perfil Responsable. En cambio, se coincide en que la motivación para visitar monumentos se relaciona con el factor Responsabilidad, no obstante, también se encontró en dicho estudio que esta se relacionaba con la Apertura y una relación de la misma con la Extraversión y la Amabilidad. Se ha comprobado que no todas las personalidades se relacionan con todas las motivaciones, en concreto, la personalidad Neurótica no se vincula de forma significativa a ninguna motivación turística. Semejante tendencia cual podría ser atribuible a las propias características diferenciales de ambos estudios ya que la investigación de Ojeda (2015) se vincula al estudio de la modalidad de turismo de ocio y, específicamente, en un destino de sol y playa como Gran Canaria. En cambio, en este estudio se han diversificado las modalidades de destino turístico, así como el origen geográfico de los propios turistas y de sus elecciones. Así, en nuestro estudio, el rasgo Extravertido no correlaciona significativamente con las motivaciones hedonistas y de descanso; la Apertura no se relaciona con la motivación hedonista y de descanso, ni monumental y gastronómica, ni con la socio-relacional y tampoco con los factores de conveniencia y utilitarios; el perfil propio de Amabilidad se relaciona prioritariamente con la búsqueda de nuevas experiencias y no con el resto y, finalmente, el rasgo de personalidad calificado como Responsabilidad, finalmente, se asocia a las motivaciones monumentales y gastronómicas, aunque con un valor bajo, y no correlaciona con motivos sociorrelacionales ni de conveniencia o utilitarios.

En un sentido análogo, se valora la psicología particular del turista como uno de los principales factores que determina la motivación para la elección de destinos, de modo que en modalidades de turismo cultural y, específicamente en quienes visitan ciudades Patrimonio de la Humanidad, se revela que sean claves factores como la Apertura a la experiencia, asociada según Ter Laak (1996) a Cultura, Intelecto, "Intelectancia": fantasía, estética, sentimientos, acciones, ideas y valores, y la calificada como personalidad alegre en el estudio emprendido por Carrillo, Pulido y Mudarra (2019). Asimismo, en destinos patrimoniales, Prada y Pensatez (2017) evidencian que la dimensión cultural de la motivación es la más importante de modo que concluyen que el turista cultural puro se impulsa por la búsqueda de una experiencia cultural de calidad (Prada et al., 2017).

Por lo que respecta a las motivaciones para viajar Guapi, Moreno y Cherrez (2020) apelan a las motivaciones que guían las experiencias de la modalidad de turismo experiencial, siendo fundamentales la búsqueda de experiencias y el afán de exploración y podría vincularse a rasgos de personalidad propios de rasgos como la Apertura a la experiencia, lo cual es coincidente con los hallazgos de nuestro estudio. Su propensión a tomar decisiones relacionadas con la experimentación de sensaciones nuevas y la innovación con predominio del pensamiento creativo son rasgos personológicos característicos que guían la motivación turística. Asimismo, al evaluar la satisfacción turística percibida se ha comprobado la vinculación con rasgos de personalidad como Apertura y Extraversión relacionadas con la percepción del bienestar (véase Steel et al., 2008).

En este estudio se ha confirmado que la satisfacción turística correlaciona con la motivación socio-relacional, monumental y gastronómica y con la búsqueda de nuevas experiencias. En este sentido, Devesa et al. (2010) comprobaron que los visitantes con motivación monumental expresaban sentir mayor satisfacción de conservación del patrimonio, mientras que los turistas gastronómicos y culturales acerca de las visitas guiadas, el tráfico, la profesionalidad de la hostelería, la oferta de alojamiento, etc. A su vez, López-Guzmán, Pérez Gálvez y Muñoz-Fernández (2018) identifican una dimensión multimotivacional de tales turistas que incluye motivos de elección y satisfacción como la búsqueda 
de desconexión con lo cotidiano, estando relacionada la motivación turística con la satisfacción con la visita y con la lealtad al destino turístico.

De acuerdo con uno de los objetivos específicos planteados relativo al análisis de las diferencias en motivación y en satisfacción turística en función de variables sociodemográficas, tales como el sexo, la edad y el nivel de estudios, se ha constatado que no existen diferencias en función del sexo. Sin embargo, las motivaciones turísticas difieren según la edad, no sucediendo lo mismo con la satisfacción turística. Por otro lado, no se han obtenido diferencias en las motivaciones turísticas en función del nivel de estudios. Así, las inferencias que se pueden realizar a partir del presente estudio son que el nivel de estudios no da lugar a diferencias en las motivaciones turísticas, por tanto, es una variable independiente que no nos influye necesariamente en la elección de un determinado destino turístico. Por el contrario, la edad influye sobre nuestras preferencias puesto que a través de ella se encuentran diferencias en las motivaciones turísticas de índole socio-relacional y la búsqueda de nuevas experiencias. En relación con ello, Moreno Gil et al. (2012) habían destacado que los menores de 34 años se motivan por la diversión y las personas mayores de 54 años por la interacción social. A su vez, Ojeda (2015) afirmó que, a medida que nos hacemos mayores, le damos más importancia a las motivaciones familiares y de descanso y menos a disfrutar de la experiencia.

En cuanto a las limitaciones del presente estudio se podría aludir al tamaño muestral, que convendría ampliar, así como diversificar la procedencia de los participantes del marco geográfico de análisis, dada la dificultad de extrapolar los resultados que han de ser circunscritos a las características y representatividad de la muestra seleccionada. Del mismo modo, otra limitación es la relativa a la desigual distribución por sexo de los participantes. Asimismo, es pertinente señalar también el carácter transversal del estudio, que impide el establecimiento de relaciones de causalidad entre las variables estudiadas. Por último, la extensión total de los cuestionarios aplicados pudo haber provocado fatiga en los participantes y ha de tenerse en cuenta el posible sesgo de deseabilidad social, lo cual nos hace ser cautos en la interpretación de los resultados. No obstante, independientemente de ello, este tipo de resultados, circunscritos a un determinado destino turístico y perfil de turista, podría resultar de interés en las estrategias de diseño de productos turísticos y la comercialización de los servicios turísticos.

En su conjunto, se valora la importancia de avanzar en el conocimiento de la aplicabilidad del estudio de la personalidad en el análisis comprehensivo del comportamiento turístico, que está multideterminado, y que podría resultar de interés en términos de oferta y demanda turística.

Aun siendo escasos los trabajos empíricos en los que se inciden en esta cuestión (véase Gretzel et al., 2004; Laesser y Zehrer, 2012; Medina-Ojeda et al., 2019; Tan y Tang, 2013). De estos hallazgos se pueden derivar diversas implicaciones prácticas vinculadas a la propia oferta turística ya que supondría segmentar los mercados atendiendo, junto a otras muchas variables determinantes, a la dimensión psicosocial que se asocia a aspectos motivacionales, así como asociados a la propia satisfacción turística. Desarrollar estrategias promocionales de destinos y las propias actividades de ocio adaptadas a tales perfiles resultaría de interés incardinadas en un plan de marketing global desde el que se contemple la interrelación de factores determinantes del comportamiento turístico.

A nivel prospectivo, ha de promoverse la realización de investigaciones en las que se analice de forma más profunda cómo influye la personalidad sobre la elección de destino turístico, así como sobre las motivaciones para viajar y la propia satisfacción turísticas. Esta cobra un renovado sentido en una sociedad digitalizada en la que el comportamiento de los consumidores en materia de viajes puede verse influido por la tendencia al uso de los medios de comunicación social vinculados al proceso de construcción de la identidad, así como a la tendencia a la búsqueda de aprobación social (Carneiro y Ferreira, 2019), máxime en personas con conflictos identitarios y una tendencia al exhibicionismo digital (Álvarez y Moral, 2020; Torre, 2020). Tales factores psicosociales de interés motivan el comportamiento social humano, específicamente en materia turística.

\section{Bibliografía}

Abbate, C. S., \& Nuovo, S. D. 2013. Motivation and personality traits for choosing religious tourism. A research on the case of Medjugorje. Current Issues in Tourism, 16(5), 501-506. doi: $10.1080 / 13683500.2012 .749844$

Ariffin, A. A. M., Ahmad, A. H., \& Ishak, N. K. 2008. Corporate Meeting Destination Choice: The Influences of Consumption Value, Organizational Structure and Personality. International Journal of Hospitality \& Tourism Administration, 9(4), 313-326. doi: 10.1080/15256480802427248 
Andrade, M. 2012. Los factores personales como variables determinantes en la formación de la imagen del destino. Especial referencia a las motivaciones turísticas. Teoría y Praxis, (11), 10-39.

Bigné, J. E., Sánchez, M. I., \& Sánchez, J. 2001. Tourism image, evaluation variables and after purchase dimensions. Tourism Management, 22, 127-133. doi: 10.1016/S0261-5177(01)00035-8

Carrillo, I., Pulido, J. I., \& Mudarra, A. B. 2019. Caracterización del turista en Ciudades Patrimonio de la Humanidad: el casi de Úbeda y Baeza. Boletín de la Asociación de Geógrafos Españoles, 81, 2746, 1-25. doi: 10.21138/bage.2746

Chi, C.G.Q., \& Qu, H. 2008. Examining the structural relationships of destination image, tourist satisfaction and destination loyalty: An integrated approach. Tourism Management, 29, 624-636. doi: 10.1016/j.tourman.2007.06.007

Chon, K. S. 1992. Self-image/destination image congruity. Annals of Tourism Research, 19(2), 360-363. doi: 10.1016/0160-7383(92)90090-C

Cloninger, S.C. 2003. Teorías de la personalidad. México: Pearson Education.

Colvin, M., \& Rutland, F. 2008. Is Maslow's Hierarchy of Needs a Valid Model of Motivation. Louisiana Tech University. Recuperado de http://www.business.latech.edu/

Costa, P. T., \& McCrae, R. R. 1980. Still stable after all these years: Personality as a key to some issues in adulthood and old age. Life Span Development and Behavior, 3, 65-102.

Devesa, M., \& Palacios, A. 2005. Predicciones en el nivel de satisfacción percibida por los turistas a partir de variables motivacionales y de valoración de la visita. Información Comercial Española, 821, 241-255. doi: 10.32796/ice.2005.821.768

Devesa, M., Laguna, M., \& Palacios, A. 2008. Un modelo estructural sobre la influencia de las motivaciones de ocio en la satisfacción de la visita turística. Revista de Psicología del Trabajo y de las Organizaciones, 24(2), 253-268.

Devesa, M., Laguna, M. \& Palacios, A. 2010a. The role of motivation in visitor satisfaction: Empirical evidence in rural tourism. Tourism Management, 31, 547-552. doi: 10.1016/j.tourman.2009.06.006

Devesa, M., Laguna, M., \& Palacios, A. 2010b. Motivación, satisfacción y lealtad en el turismo: el caso de un destino de interior. Revista Electrónica de Motivación y Emoción. Monográfico de la Motivación del Consumidor XIII (35 y 36), 169-190.

Digman, J. M., \& Takemoto-Chock, N. K. 1981. Factors in the natural language of personality: Re-analysis, comparison, and interpretation of six major studies. Multivariate Behavioral Research, 16(2), 149-170. doi:10.1207/ s15327906mbr1602_2

Dumazedier, J. 1974. Sociologie empirique du loisir. Critique et contracritique de la civilisation du loisir. Paris: Du Seuil.

Garcés, S., Pocinho, M., Neves, S., \& Rieber, M. S. 2018. Positive Psychology \& Tourism. Tourism \& Management Studies, 14(3), 41-51.

García, J. S. M. 1997. Psicosociología del ocio y el turismo. Psicothema, 9(3), 689-691.

Goldberg, L. R. 1981. Language and individual differences: The search for universals in personality lexicons. Review of Personality and Social Psychology, 2(1), 141-165.

González, J. 2018. La actividad turística como fenómeno complejo. Una visión Alternativa. Controversias Y Concurrencias Latinoamericanas, 10(16), 111-117. Recuperado a partir de http://sociologia-alas. org/CyCLOJS /index.php/CyC/article/view/58

Gountas, J., \& Gountas, S. 2007. Personality orientations, emotional states, customer satisfaction, and intention to repurchase. Journal of Business Research, 60(1), 72- 75. doi: 10.1016/j.jbusres.2006.08.007

Graziano, W. G., \& Tobin, R. M. 2009. En M. R. Leary \& R. H. Holey (eds.). Agreeableness. Handbook of individual differences in social behavior (pp. 46-61). New York: Guilford.

Gretzel, U., Mitsche, N., Hwang, Y.H., \& Fesenmaier, D.R. 2004. Tell me who you are and I will tell you where to go: use of travel personalities in destination recommendation systems. Information Technology \& Tourism, 7(1), 3-12.

Guapi, F. I., Moreno, J. S., \& Cherrez, R. C. 2020. El turismo experiencial, deportes y juegos tradicionales: nueva perspectiva para el desarrollo turístico local. Revista Arbitrada Interdisciplinaria Koinonía, 5(10), 904-919.

Kalmus, V., Realo, A., \& Siibak, A. 2011. Motives for Internet use and their relationships with personality traits and socio-demographic factors. Trames, 15(4), 385- 403. doi: 10.3176/tr.2011.4.04

Kozak, M., \& Rimmington, M. 2000. Tourist satisfaction with Mallorca, Spain, as an off-season holiday destination. Journal of Travel Research, 38(3), 260-269. doi: 10.1177/004728750003800308 
Laesser, C., \& Zehrer, A. 2012. Tell me who you think you are and I tell you how you travel: Exploring the viability of market segmentation by means of travelers' stated personality: Insights from a mature market (Switzerland). Tourism Analysis, 17(3), 285-298. doi: 10.3727/108354212X13412775927781

Laguna, M., \& Palacios, A. 2009. La calidad percibida como determinante de tipologías de clientes y su relación con la satisfacción: Aplicación a los servicios hoteleros. Revista Europea de Dirección y Economía de la Empresa, 18(3), 189-212. ISSN 1019-6838

Lee, Y. C. 2013. The influence of personality traits, health knowledge, and product attributes on intent to purchase Taiwan's healthcare tourism products. Social Behavior and Personality, 41(3), 395-410. doi: 10.2224/sbp.2013.41.3.395

Leung, R., \& Law, R. 2010. A Review of Personality Research in the Tourism and Hospitality Context. Journal of Travel \& Tourism Marketing, 27(5), 439-459. doi: 10.1080/10548408.2010.499058

Lin, Y., Kerstetter, D., Nawijn, J., \& Mitas, O. 2014. Changes in emotions and their interactions with personality in a vacation context. Tourism Management, 40, 416- 424. doi: 10.1016/j.tourman.2013.07.013

López, J. L., \& Daniel, A. 2019. Emotividad positiva, el beneficio esperado de hacer turismo. Realidad, Tendencias y Desafíos en Turismo, 17(2), 27-44.

López-Guzmán, T., Pérez Gálvez, J. C., \& Muñoz-Fernández, G. A. 2018. Satisfaction, motivation, loyalty and segmentation of tourists in World Heritage cities. PASOS Revista De Turismo Y Patrimonio Cultural, 16(1), 73-86. doi: 10.25145/j.pasos.2018.16.005

Maslow, A. H. 1943. A theory of human motivation. Psychological Review, 50(4), 370-396. doi: 10.1037/ h0054346

McCrae, R. R., \& Costa, P. T. 2003. Personality in Adulthood: A Five-factor Theory Perspective. Nueva York: The Guilford Press.

McCrae, R. R., \& Sutin, A. R. 2009. Openness to Experience. En M. R. Leary and R. H. Hoyle (Eds.) Handbook of individual differences in social behavior (pp. 57- 273). New York: Guilford.

Medina-Ojeda, N., Martín-Santana, J. D., \& Medina-Muñoz, D. R. 2019. La personalidad del turista como criterio de segmentación de destinos de sol y playa: una aplicación al destino Gran Canaria. Cuadernos De Turismo, 1(44), 247-275. doi: 10.6018/turismo.44.404831

Moral, M. V. 2009. Ocio bifronte en una sociedad postindustrial: análisis psicosociológico de las tendencias emergentes. Boletín de Psicología, 96, 47-65.

Moreno Gil, S., Beerli-Palacio, A., \& León, J. D. 2012. Entender la imagen de un destino turístico: factores que la integran y la influencia de las motivaciones. Criterio Libre, 10(16), 115-142.

Munné, F. 1980. Psicosociología del tiempo libre: Un enfoque crítico. México: Trillas.

Norman, W. T. 1963. Toward an adequate taxonomy of personality attributes: replicated factors structure in peer nomination personality ratings. Journal of Abnormal and Social Psychology, 66(6), 574-583. doi:10.1037/ h0040291

Ojeda, N. M. 2015. Influencia de la personalidad en el comportamiento del turista: una aplicación empírica en Gran Canaria. Tesis Doctoral: Universidad de Las Palmas de Gran Canaria. Recuperado de https://accedacris.ulpgc.es/bitstream/ 10553/17411/3/0724523_00000_0000.pdf

Olague, J. T., Flores, C. A., \& Garza, J. B. 2017. El efecto de la motivación de viaje sobre la satisfacción del turista a través de las dimensiones de la imagen del destino: EL caso del turismo urbano de ocio a Monterrey, México. Investigaciones Turísticas (14), 109-129 http://dx.doi.org/10.14461/INTURI2017.14.06

Oliver, R. L. 1997. Satisfaction: A Behavioural Perspective on the Consumer. Boston: McGraw-Hill.

Organización Mundial del Turismo 1994. Concepts, definitions and classifications for tourism statistics. Madrid: UNWTO.

Organización Mundial del Turismo 2019a. Definiciones de turismo de la OMT. OMT, Madrid, DOI: https://doi.org/10.18111/9789284420858.

Organización Mundial del Turismo 2019b. Panorama OMT del turismo internacional. Madrid: UNWTO.

Pazos, A. 2017. La evolución del turismo en España. Trabajo de Fin de Grado. Universidad de Valladolid. Recuperado de https://uvadoc.uva.es/bitstream/handle/10324/24042/TFG- N.634.pdf;jsessionid=AA 983520765789809BFAFD7360045682? sequence=1

Pearce, P. L. 1982. Perceived changes in holiday destinations. Annals of Tourism Research, 9(2), 145-164. doi: 10.1177/004728758302200163

Pearce, P. L. 1987. Psychological studies of tourist behaviour and experience. Australian Journal of Psychology, 39(2), 173-182. doi: 10.1080/00049538708259046

Pinillos, J. L. 1990. El turismo como hecho psicológico. Papers de Turisme, (2), 5-13.

Prada, J., \& Pesantez, S. 2017. Satisfacción y motivación en destinos culturales: Tipología de los turistas atraídos por el patrimonio inmaterial en Cuenca (Ecuador). Diálogo Andino, 52, 77-91. 
Prada, J., Armijos, D., Crespo, A., \& Torres, L. 2017. Destinos turísticos culturales y satisfacción: diferencias en la intención de regreso. Estudios y Perspectivas en Turismo, 26(4), 864-883.

Roberts, B. W., Jackson, J. J., Fayard, J. V., Edmonds, G., \& Meints, J. 2009. En M. Leary \& R. Hoyle (Eds.). Conscientiousness. Handbook of Individual Differences in Social Behavior (pp. 257-273). New York: Guilford Press.

Steel, P., Schmidt, J., \& Shultz, J. 2008. Refining the relationship between personality and Subjective well-being. Psychological Bulletin 134(1), 138-161.

Tan, W., \& Tang, C. 2013. Does personality predict tourism information search and feedback behaviour? Current Issues in Tourism, 16(4), 388-406.

Ter Laak, J. 1996. Las cinco grandes dimensiones de la personalidad. Revista de Psicología de la PUCP, XIV, 2, 129-181.

Torre, F. 2020. Comportamiento turístico en Millenials y exhibicionismo digital. Trabajo Fin de Grado. Gijón: Universidad de Oviedo.

Tupes, E. C., \& Christal, R. E. 1961. Recurrent personality factors based on trait ratings. Journal of Personality, 60(2), 225-251. doi:10.1111/j.1467-6494.1992. tb00973.x

Widiger, T. A. 2009. Neuroticism. En M. R. Leary \& R. H. Hoyle (Eds.) Handbook of Individual Differences in Social Behavior (pp. 129-146). New York: The Guilford Press.

Wilt, J., \& Revelle, W. 2009. Extraversion. En M. Leary \& R. Hoyle (Eds). Handbook of individual differences in social behavior (pp. 27-45). New York: Guilford.

Yoon, Y., \& Uysal, M. 2005. An examination of the effects of motivation and satisfaction on destination loyalty: a structural model. Tourism Management, 26(1), 45- 56. doi: 10.1016/j.tourman.2003.08.016

Yuksel, A., Yuksel, F., \& Bilim, Y. 2010. Destination attachment: Effects on customer satisfaction and cognitive, affective and conative loyalty. Tourism Management, 31(2), 274-284. doi:10.1016/j. tourman.2009.03.007 